\title{
The Role of the $\mathrm{PI}(3,5) \mathrm{P}_{2}$ Kinase TbFab1 in Endo/Lysosomal Trafficking in Trypanosoma brucei.
}

\author{
Julia K. Gilden ${ }^{1}$, Khan Umaer ${ }^{2}$, Emilia Kruzel ${ }^{2}$, Oliver Hecht ${ }^{1}$, Renan O. Correa ${ }^{1}$, John M. \\ Mansfield ${ }^{1}$, and James D. Bangs ${ }^{2 *}$
}

${ }^{1}$ Department of Bacteriology, University of Wisconsin-Madison, Madison, WI, USA

${ }^{2}$ Department of Microbiology Immunology, School of Medicine and Biomedical Sciences, University at Buffalo (SUNY), Buffalo, NY, USA

* Corresponding Auther: James D. Bangs, Ph.D.

Department of Microbiology \& Immunology Jacobs School of Medicine \& Biomedical Sciences University at Buffalo, 138 Farber Hall 3435 Main Street Buffalo, NY 14214

Julia K. Gilden: $\quad$ julia.gilden@gmail.com Khan Umaer: $\quad$ khanumae@buffalo.edu Emilia Kruzel: $\quad$ emiliakr@buffalo.edu Oliver Hecht: ohecht@wisc.edu Renan O. Correa: renanocorrea@gmail.com John M. Mansfield: john.mansfield@wisc.edu James D. Bangs: jubangs@buffalo.edu

Key Words: Trypanosome, $\mathrm{PI}(3,5) \mathrm{P}_{2}$, Endosome, Lysosome, Invariant Surface Protein 6 Running Title: $\mathrm{PI}(3,5) \mathrm{P}_{2}$ function in African trypanosomes

Abbreviations: MVB: Multivesicular Body, ESCRT: Endosomal Sorting Complex Required for Transport, ILV: Intraluminal Vesicle, LE: Late Endosome, ISG: Invariant Surface Glycoprotein, PIP: Phosphoinositide, BSF: Bloodstream Form, TL: Tomato Lectin, 


\section{ABSTRACT (200)}

Protein trafficking through endo/lysosomal compartments is critically important to the biology of the protozoan parasite Trypanosoma brucei, but the routes material may take to the lysosome, as well as the molecular factors regulating those routes, remain incompletely understood.

Phosphoinositides are signaling phospholipids that regulate many trafficking events by recruiting specific effector proteins to discrete membrane subdomains. In this study, we investigate the role of one phosphoinositide, $\mathrm{PI}(3,5) \mathrm{P}_{2}$ in $T$. brucei. We find a low steady state level of $\mathrm{PI}(3,5) \mathrm{P}_{2}$ in bloodstream form parasites comparable to that of other organisms. RNAi knockdown of the putative $\mathrm{PI}(3) \mathrm{P}-5$ kinase TbFab1 decreases the $\mathrm{PI}(3,5) \mathrm{P}_{2}$ pool leading to rapid cell death. TbFab1 and $\mathrm{PI}(3,5) \mathrm{P}_{2}$ both localize strongly to late endo/lysosomes. While most trafficking functions were intact in TbFab1 deficient cells, including both endocytic and biosynthetic trafficking to the lysosome, lysosomal turnover of an endogenous ubiquitinylated membrane protein, ISG65, was completely blocked suggesting that TbFab1 plays a role in the ESCRT-mediated late endosomal/multivesicular body degradative pathways. Knockdown of a second component of $\mathrm{PI}(3,5) \mathrm{P}_{2}$ metabolism, the $\mathrm{PI}(3,5) \mathrm{P}_{2}$ phosphatase TbFig4, also resulted in delayed turnover of ISG65. Together, these results demonstrate an essential role for $\mathrm{PI}(3,5) \mathrm{P}_{2}$ in the turnover of ubiquitinylated membrane proteins and in trypanosome endomembrane biology.

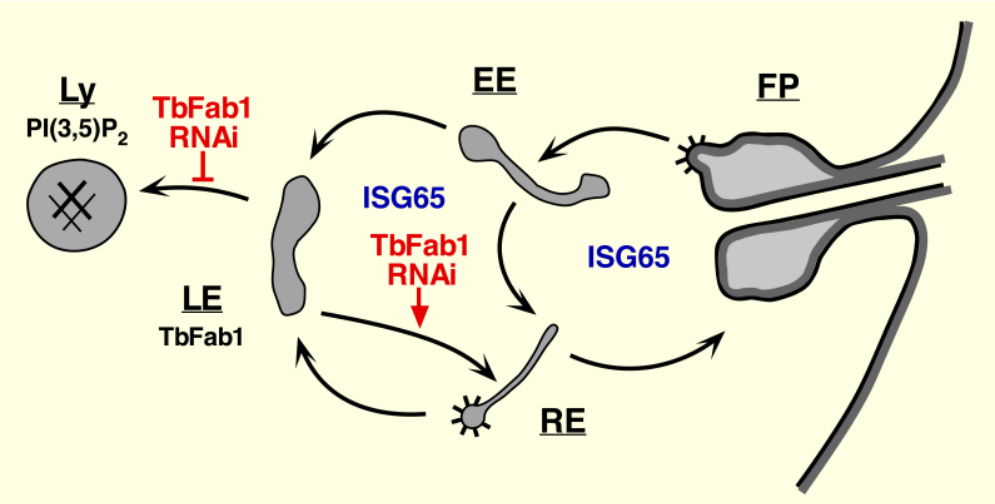

ISG65 recycles through endosomal compartments and is ultimately degraded in the lysosome. Depleting $\mathrm{PI}(3,5) \mathrm{P}_{2}$ rescues ISG65 by blocking lysosomal delivery and/or enhancing retrieval from the late endosome. 


\section{1. Introduction}

2 Trypanosoma brucei subspecies are parasitic protozoa transmitted by tsetse flies to a

3 wide variety of mammalian hosts, causing Human African Trypanosomiasis (HAT or African

4 Sleeping Sickness) in man and nagana in cattle and other livestock. Untreated infections can

5 persist for months or years before the host ultimately succumbs to the disease. The parasite

6 lives free in the bloodstream and tissues of the infected mammal and avoids the host adaptive

7 immune system by a process of antigenic variation [1, 2]. Essential to survival in this hostile

8 environment is the parasite's endolysosomal system. Endocytosis is accelerated in the

9 bloodstream form relative to other developmental stages [3, 4], and contributes to parasite

10 nutritional needs, as well as evasion of humoral immune responses [3, 5-8]. Paradoxically, the

11 lysosome also represents a point of vulnerability for T. brucei, and is a target of both the human

12 innate immune system and some chemotherapeutic strategies [9, 10].

13 The path of endocytic cargo to the lysosome for degradation in T. brucei is generally

14 similar to that of other model eukaryotes in that subcompartments are marked by characteristic

15 small regulatory GTPases called Rabs. Cargo is endocytosed from the flagellar pocket, a

16 specialized subdomain of the plasma membrane, in clathrin-coated vesicles [3, 4], which

17 subsequently fuse with TbRab5-postive early endosomes [11,12]. From there, endocytosed

18 material can be sorted either to TbRab11-positive recycling endosomes for return to the cell

19 surface $[11,13]$, or to TbRab7-positive late endosomes for delivery to the single terminal

20 lysosome and subsequent degradation $[11,14,15]$. Late endosomal material can also be

21 diverted for recycling, presumably via the recycling endosome [11].

22 In yeast and animals, transmembrane proteins can be turned over by trafficking through

23 a specialized late endosome called the multivesicular body (MVB), and progress through this

24 organelle is mediated by the stepwise assembly of ESCRT (E्ndosomal Sorting $\underline{\text { Complex }}$

25 Required for Transport) proteins [16-18]. Such proteins are marked for turnover by 
26 monoubiquitinylation of lysine residues in their cytoplasmic domains, and once in an endosomal

27 compartment such ubiquitinylated species are aggregated laterally by ESCRT0 and ESCRT1

28 protein complexes. Areas of aggregation then invaginate via the action of ESCRT2 complex,

29 and ESCRT3 complex is responsible for subsequent scission into intraluminal vesicles (ILVs).

30 Ultimately the spent ESCRT machinery is disassembled by an ATPase, Vps4, which provides

31 the sole energetic input to the system [19].

32 Characteristic MVBs with distinct ILVs are not morphologically obvious in trypanosomes,

33 but clear orthologues of the ESCRT1-3 machinery are evident in the T. brucei genome, two of

34 which (TbVps23 and TbVsp4) have been characterized [15, 20]. Both colocalize with TbRab7

35 to the late endosome (LE) consistent with MVB-type functions [15]. Ubiquitinylation has also

36 been shown to be important for turnover of two trypanosome invariant surface glycoproteins,

37 ISG65 and ISG75 [21-23]. Both are Type I membrane glycoproteins with lysine residues in their

38 cytoplasmic domains that are subject to ubiquitinylation. Initially, based on RNAi silencing of

39 TbVps23, it was argued that the classic ESCRT pathway was responsible for ubiquitin

40 dependent lysosomal targeting and degradation of ISG65 [20]. However, we were unable to

41 reproduce that finding, and in contrast found that silencing of TbVps4 actually accelerated

42 turnover of ISG65, as did silencing of TbRab7 [15]. These results suggest that the role of the

43 trypanosomal ESCRT machinery in regard to ISGs is rescue from lysosomal targeting by

44 retrieval and recycling from the late endosome. Whether this is dependent on ubiquitinylation is

45 unknown. Such a role for ESCRT, which has many cellular functions besides MVB formation, is

46 not without precedent - ESCRT is critical for recycling of EGF receptor in some mammalian cell

47 lines [24].

48 These potentially important distinctions between the endosomal pathways in T. brucei

49 and other eukaryotes has sparked interest in studying other mediators of the pathway, and in

50 trying to understand the complete network of routes that endocytosed material may take to the

51 lysosome or back to the flagellar pocket. One such mediator is the signaling phospholipid, 
52 phosphatidylinositol $(3,5)$ bisphosphate $\left(\mathrm{PI}(3,5) \mathrm{P}_{2}\right)$. Like other phosphoinositides (PIPs),

$53 \mathrm{PI}(3,5) \mathrm{P}_{2}$ sits in the cytoplasmic leaflet of membranes and recruits effector proteins to carry

54 outlocalized functions on those membranes. This lipid is produced when a kinase, Fab1p in

55 yeast or PIKfyve in mammals, phosphorylates PI3P on the 5 position of the inositol ring $[25,26]$.

56 Depletion of the kinase in many systems leads to disregulation of the vacuole or lysosome,

57 including loss of acidification, morphological changes, disrupted trafficking of ubiquitinylated

58 cargo, and severe growth defects [25-32].

59 In this study we examine the role of $\mathrm{PI}(3,5) \mathrm{P}_{2}$ in $T$. brucei by disrupting the enzymes that

60 control its metabolism. We find that while steady-state levels of $\mathrm{PI}(3,5) \mathrm{P}_{2}$ are very low in $T$.

61 brucei, as in other eukaryotes, the lipid is profoundly important for turnover of ISG65 and for

62 parasite survival.

\section{2. Materials and methods}

65 2.1 Parasite cultures Lister 427 BSF parasites were grown in HMI9 media with $20 \%$ fetal 66 bovine serum [33], and with antibiotics as appropriate for maintaining transgenes. Tetracycline

67 inducible cell lines, produced in the single-marker BSF background [34], were grown in HMI9

68 supplemented with tet-free fetal bovine serum (Atlanta Biologicals). For experiments, cells were

69 harvested at mid-late log phase (BSF, $\left.0.5 \times 10^{6}-10^{6}\right)$.

712.2 RNAi and tagging constructs dsRNA against TbFab1 and TbFig4 was performed using

72 the dual promoter tetracycline-responsive p2T7i vector [35]. 930 (TbFab1) or 700 (TbFig4)

73 base pair regions of the genes were PCR amplified with flanking 5' BamHI and 3' Hindlll cloning

74 sites for insertion into p2T7i. Primers were (restrictions sites in lower case): TbFab1 5':

75 ggatccTTGGAAGTTGTGAGGGCTATTT; TbFab1 3': aagcttAATAAGCGTGTCTGGGTAACG,

76 TbFig4 5': ggatccACGGTATCCACTTCCAGTGC, TbFig4 3': 
77 aagcttTTGGCAAGAGTGGAGCTTTT. The ENTH domain of yeast Ent3p (plasmid pFL670,

78 [36]) was a gift of Professor Sylvie Friant (University of Strasbourg). 5' Acll and 3' EcoRI sites

79 were added by PCR and the product was cloned into the Clal and EcoRI sites of the constitutive

$80 \mathrm{pXS6}{ }^{3 \times \mathrm{HA}}$ vector (neomycin selection) [14], generating an in frame C-terminal 3xHA-tag fusion.

81 The plasmid was linearized with Notl for insertion into the rDNA locus. In situ chromosomal C-

82 terminal HA-tagging of TbFab1 was performed using the pXS6 ${ }^{3 \times H A}$ vector. 454 bp at the 3' end

83 of the open reading frame and $539 \mathrm{bp}$ of the immediate 3' UTR were amplified and sequentially

84 cloned into pXS6 ${ }^{3 \times H A}$

85 using the 5' HindIII/3' EcoRI and 5' Pacl/3' Sacl sites, respectively. Primers were: TbFab1 ORF

86 5', aagcttGAACCGTGTAGCGCGTGAG; TbFab1 ORF 3',

87 gaattcAGCCAAACTGCATGAATCCCCT 3' UTR 5', ttaattaaCACACCGTCAACACCACCAG; 3’

88 UTR 3', gagctcGTTTAACTCACTTCCTCGTTGCCG. As experimental contingency indicated

89 that robust detection of the tagged protein required tagging of both alleles, the construct was

90 prepared with both neomycin and puromycin resistance cassettes. Both plasmids were

91 linearized with HindIII and Sacl for chromosomal tagging. The TY-Rab7 in situ tagging

92 construct has been described previously [14]. All constructs were verified by sequencing.

$94 \quad 2.3$ Transfection and cloning of cell lines Linearized plasmids were transfected using Mirus

95 Ingenio transfection solution on an Amaxa Nucleofector. Transfected parasites were then

96 plated by limiting dilution and cultured with appropriate antibiotics to select for stably transfected

97 clones.

98

99 2.4 PIP extraction and head group analysis Trypanosomes were cultured overnight in

100 inositol deficient media DMEM (US Biological, Salem MA) supplemented with 36 mM sodium

101 bicarbonate, $1 \mathrm{mM}$ hypoxanthine, $50 \mu \mathrm{M}$ bathocuproine, $1.5 \mathrm{mM}$ cysteine, $0.2 \mathrm{mM}$ 2- 
102 mercaptoethanol, $1 \mathrm{mM}$ pyruvate, $0.16 \mathrm{mM}$ thymidine, 20\% FBS, and $100 \mathrm{U} / \mathrm{mL}$ penicillin and

103 streptomycin in the presence of $10 \mu \mathrm{Ci} / \mathrm{mL}\left[{ }^{3} \mathrm{H}\right]$ myo-inositol (American Radiolabeled Chemical,

104 St Louis, MO; 30-80 Ci/mmol). To assess the effect of TbFab1 knockdown on $\mathrm{PI}(3,5) \mathrm{P}_{2}$

105 production, tetracycline was included throughout the labeling period. Starting densities were

$1062 \times 10^{5} / \mathrm{ml}$ for control cells and $4 \times 10^{5} / \mathrm{ml}$ for knockdown cells, which have impaired growth

107 compared to controls. Labeled cells were then washed $3 x$ in buffered saline with glucose and

108 final pellets were flash frozen in a dry ice/ethanol bath. Isolation of glyceroPIP head groups was

109 based on the protocol described in [37]. Pellets were extracted in methanol:chloroform:2.4 M

$110 \mathrm{HCl}(125: 250: 10)$ and lipids were back-extracted in methanol:1M HCl:chloroform (235:245:15).

111 After drying under $\mathrm{N}_{2}$, lipids were resuspended in fresh methylamine reagent (methanol:40\%

112 methylamine:1-butanol at $1: 1: 0.1)$ and incubated at $50^{\circ} \mathrm{C}$ for 1 hour for deacylation. Head

113 groups and fatty acids were separated by extraction with butanol/petroleum ether/ethyl formate

114 (20:4:1). Purified glyceroylPIP (gPIP) head groups were then dried and resuspended in $10 \mathrm{mM}$

$115\left(\mathrm{NH}_{4}\right)_{2} \mathrm{PO}_{4} \mathrm{pH} 3.8$ for analysis. gPIPs were separated by anion exchange HPLC on a Beckman

116 System Gold (Beckman Coulter Life Sciences, Indianapolis, IN) using an analytical 25-cm

117 Partisil 5 SAX column (Hichrom, Reading, UK) and the following gradient of $\left(\mathrm{NH}_{4}\right)_{2} \mathrm{PO}_{4}, \mathrm{pH} 3.8$

118 at a flow rate of $1 \mathrm{~mL}$ per minute: $10 \mathrm{mM}$ for $5 \mathrm{~min}, 10-125 \mathrm{mM}$ over $40 \mathrm{~min}$, and $125 \mathrm{mM}$ to 1.0

119 M over 10 min. Fractions were collected every 0.7 minutes, and $\left[{ }^{3} \mathrm{H}\right] \mathrm{gPIPs}$ were detected by

120 liquid scintillation counting. To generate $\mathrm{PIP}_{2}$ standards S. cerevisiae cells were labeled for 20

121 hours in inositol-free YPD media with $10 \mu \mathrm{Ci} / \mathrm{mL}\left[{ }^{3} \mathrm{H}\right]$ myo-inositol. Labeled cells were washed,

122 resuspended and incubated in hyperosmotic zymolyase buffer containing 30 mM DTT for 15

123 minutes. Next, cells were centrifuged and resuspended in zymolyase buffer with $67 \mathrm{U} / \mathrm{mL}$

124 zymolyase and incubated at $30^{\circ} \mathrm{C}$ for $40^{\prime}$ with shaking. Resulting spheroplasts were centrifuged, 125 snap frozen, and PIPs were extracted and treated as described above. 
1272.5 qRT-PCR RNA was extracted using a Qiagen RNEasy Mini kit with on-column DNase

128 digestion (Qiagen, Valencia, CA). cDNA was produced from 1-3 $\mu$ g RNA using Superscript III

129 Reverse Transcriptase (Thermo Fisher Scientific, Waltham, MA) and anchored oligo-dT (IDT)

130 according to the manufacturers instructions. cDNA was then diluted 1:10 in nuclease-free water

131 and used for qPCR analysis. qPCR was performed using Power SYBR Green Master Mix

132 (Thermo Fisher) on a BioRad CFX96 qPCR system. TbZFP3 (Tb927.3.720, nts 241-301) was

133 used as the control amplicon. All calculations and normalizations were done using native

134 Biorad software. Reactions were performed in technical triplicates, and means \pm standard

135 errors of the means (SEM) for three biological replicates are presented.

1372.6 Immunofluorescence staining and microscopy Immunofluorescence staining was

138 performed as described [14]. Briefly, cells were fixed with methanol/acetone and stained with

139 the following primary antibodies diluted in blocking buffer: rat anti-130 HA (Roche Diagnostics,

140 Indianapolis, IN), monoclonal anti-TY 1:2000 (UAB Hybridoma Facility, Birmingham, AL), rabbit

141 anti-ISG65 (gift of Professor Mark Carrington, Cambridge University, UK), and monoclonal anti-

142 p67 and rabbit anti-TbCatL [38]. AlexaFluor conjugated goat secondary antibodies (Molecular

143 Probes, Eugene OR) were used as appropriate. Stained cells were mounted in Vectashield

144 mounting medium with DAPI (Vector Laboratories, Burlingame CA). Images were acquired on a

145 Zeiss Axiolmager M2 using a 100x oil immersion objective, with exposure times $<500 \mathrm{msec}$,

146 and images were analyzed and prepared using ImageJ software (NIH).

$148 \quad 2.7$ Radiolabeling and Immunoprecipitation Metabolic labeling and immunoprecipitation

149 were performed as previously described [14]. Briefly, cells were pulsed radiolabeled in labeling

150 media for 10 minutes with $\left[{ }^{35}\right.$ S]Met/Cys (American Radiolabeled Chemicals), then chased in

151 complete pre-warmed HMI9 for the times indicated. Clarified lysates prepared in RIPA buffer 
152 and immunoprecipitated overnight at $4^{\circ} \mathrm{C}$ and resolved by SDS-PAGE. After drying, gels were

153 used for phosphorimaging and images were collected using a Typhoon FLA 9000 (GE

154 Healthcare Life Sciences) and quantitative analysis was performed in ImageJ (NIH).

2.8 Flow cytometry trafficking assays Assays measuring uptake and trafficking of labeled

157 tomato lectin were performed as previously described $[14,15]$. Binding and endocytosis were measured by incubating cells for $30^{\prime}$ at $4^{\circ} \mathrm{C}$ (binding) or $37^{\circ} \mathrm{C}$ (uptake) in serum-free HMI-9 with

$159 \quad 0.5 \mathrm{mg} / \mathrm{mL}$ BSA and $5 \mu \mathrm{g} / \mathrm{mL}$ Alexa488-conjugated tomato lectin (Molecular Probes).

160 Trafficking to the lysosome was measured by labeling cells at $4^{\circ} \mathrm{C}$ for $30^{\prime}$ in serum-free $\mathrm{HMI}-9$

161 with $0.5 \mathrm{mg} / \mathrm{mL}$ BSA and $5 \mu \mathrm{g} / \mathrm{mL}$ FITC-conjugated tomato lectin (Jackson ImmunoResearch

162 Laboratories, West Grove PA), washing, then chasing for the indicated times at $37^{\circ} \mathrm{C}$. Cells

163 were labeled with $5 \mu \mathrm{g} / \mathrm{mL}$ DAPI prior to analysis for dead-cell exclusion. Flow cytometry was

164 performed on an LSR II with FACSDiva acquisition software (BD Biosciences), and analysis 165 was done in FlowJo 8.8.7.

1672.9 ISG65 turnover Cells were incubated with cycloheximide $(100 \mu \mathrm{g} / \mathrm{ml})$ to inhibit de novo 168 protein synthesis. As indicated the lysosomal thiol protease inhibitor FMK024 (morpholinourea169 phenylalanine-homophenylalanine-fluoromethyl ketone; $20 \mu \mathrm{M}$; MP Biomedicals, Aurora, $\mathrm{OH}$ ) 170 was included prior to and during cycloheximide treatment. Whole cell lysates were fractionated 171 by SDS-PAGE, transferred using an Owl semi dry apparatus (Thermo Fisher Scientific) to 172 Immobilon-P membranes (Millipore Corp., Bedford, MA), and blocked in a 5\% milk solution.

173 Membranes were incubated $1 \mathrm{hr}$ with anti-ISG65 (1/50,000), washed, and incubated with anti174 rabbit IgG:HRP $(1 / 10,000,1 \mathrm{hr})$. Blots were stripped and reprobed with rabbit anti-BiP. The final 175 washed blots were visualized on X-ray film using Pierce SuperSignal West Pico substrate 176 (Thermo Fisher Scientific), as described elsewhere [39]. Film was digitized to 16-bit grayscale 
177 using a transparency scanner and bands quantified using the gel volume analysis function of

178 ImageJ $1.46(\mathrm{NIH}$, Bethesda, MD).

2.10 Statistical Analysis Statistical analyses were performed with Prism4 software (GraphPad

181 Software, Inc., San Diego CA). Tests and numbers of replicates are indicated in the figure 182 legends.

\section{3. Results}

1853.1 Measurement of $\mathrm{PI}(3,5) \mathrm{P}_{2}$ in bloodstream form cells In all eukaryotes studied, $\mathrm{PI}(3,5) \mathrm{P}_{2}$

186 is regulated by a multi-protein complex consisting minimally of a kinase, Fab1p (PIKfyve in 187 mammals), a phosphatase, Fig4p, and a scaffolding protein, Vac14p [40-42]. A simple BLASTP

188 search of the T. brucei reference genome (tritrypdb.org) with yeast sequences revealed clear

189 gene orthologues for each of these proteins. TbFab1 (Tb927.11.1460, E 5e-36), TbFig4

190 (Tb927.11.5490, E 6e-62) and TbVac14 (Tb927.7.3530, E 6e-29) share significant homology in

191 their enzymatic domains with their mammalian and yeast counterparts, and the domain

192 structures of all three proteins are conserved across species (Fig. 1, Fab1 \& Fig4 only). These

193 observations suggest that the metabolism of $\mathrm{PI}(3,5) \mathrm{P}_{2}$ in $T$. brucei may be similar to that in other 194 species.

$195 \mathrm{PI}(3,5) \mathrm{P}_{2}$ is typically present at very low abundance in most eukaryotes relative to other

196 PIPs. However, its synthesis in yeast can be elevated by osmotic stress [43-45]. To determine 197 whether $\mathrm{PI}(3,5) \mathrm{P}_{2}$ is present in $T$. brucei, we metabolically labeled bloodstream form (BSF) cells 198 overnight with $\left[{ }^{3} \mathrm{H}\right]$ myo-inositol, extracted and saponified all phospholipids, and analyzed the 199 resultant radiolabeled glycerol-PIP headgroups (gPIPs) by anion-exchange HPLC (Fig. 2A). 200 Equivalently labeled and prepared gPIPs from osmotically stressed yeast were analyzed in 201 parallel as chromatography standards. As expected, characteristic peaks representing 
$202 \mathrm{gPl}(3,5) \mathrm{P}_{2}$ and a related species, $\mathrm{gPl}(4,5) \mathrm{P}_{2}$, were detected at similar levels in the yeast 203 extracts $[43,45]$. The identity of the $\mathrm{gPI}(4,5) \mathrm{P}_{2}$ species was confirmed by coelution in parallel

204 chromatograms of authentic $\left[{ }^{3} \mathrm{H}\right] \mathrm{PI}(4,5) \mathrm{P}_{2}$ added to unlabeled cells and prepared and

205 fractionated identically (data not shown). A similarly strong peak representing $g \mathrm{Pl}(4,5) \mathrm{P}_{2}$ is

206 seen in the trypanosome extracts, and upon close examination a minor but reproducible peak

207 corresponding to $\mathrm{gPI}(3,5) \mathrm{P}_{2}$ is also detectable. The relative abundances of $\mathrm{gPI}(3,5) \mathrm{P}_{2}$ and

$208 \mathrm{gPI}(4,5) \mathrm{P}_{2}$ in trypanosomes are comparable to that typically observed in other organisms [42].

209 To determine whether TbFab1 is indeed responsible for production of $\mathrm{PI}(3,5) \mathrm{P}_{2}$ in

210 trypanosomes, we used an RNAi knockdown approach. We expressed a tetracycline (tet)-

211 inducible double stranded RNA construct in BSF cells. Induction for 24 hours resulted in an

$212 \sim 25 \%$ reduction in TbFab1 mRNA, as measured by qRT-PCR (\% of control 76.9 \pm 6.2 ,

213 mean \pm S.D., $n=3$ ). Despite the modest reduction, within that time period growth of parasites

214 ceased almost completely, and no recovery was observed within the first 72 hours (Fig. 2B).

215 RNAi cells were labeled with $\left[{ }^{3} \mathrm{H}\right]$ myo-inositol during the first 18 hours of tet induction and gPIPs

216 from control and knockdown cells were analyzed by HPLC (Fig. 2C). TbFab1 silencing had no

217 effect on synthesis of $\mathrm{gPI}(4,5) \mathrm{P}_{2}$, but the minor $\mathrm{gPI}(3,5) \mathrm{P}_{2}$ species was reproducibly reduced to

218 barely detectable levels. Collectively these data indicate that TbFab1 is a PI3P-5 kinase and is

219 the major kinase responsible for $\mathrm{PI}(3,5) \mathrm{P}_{2}$ production in $T$. brucei. Importantly, gross

220 morphology was unaltered at the 18hr time point, in particular there was no evidence of internal

221 vacuolization or flagellar pocket swelling as judged by differential interference contrast

222 microscopy (data not shown).

223

$224 \quad 3.2$ Localization of TbFab1 and $\mathbf{P I}(3,5) \mathbf{P}_{2}$ Fab1p in yeast and PIKfyve in mammals localize

225 to the vacuole and lysosome, respectively, and we expect similar localizations in trypanosomes.

226 To address this, we created a cell line in which both chromosomal alleles of TbFab1 were fused

227 in situ with a C-terminal 3xHA epitope tag (TbFab1:HA). Growth of this doubly tagged cell line 
228 was unaffected indicating that the tag does not impair TbFab1 function (data not shown).

229 TbFab1 is predicted to be a $156 \mathrm{kDa}$ protein, although a spliced leader trapping dataset [46]

230 indicates the use of an alternative internal trans-splice site within the TbFab1 ORF (see Fig. 1)

231 followed by an in frame start codon, which would result in a much smaller $63 \mathrm{kDa}$ protein.

232 Indeed, western blotting of TbFab1:HA lysates revealed that the vast majority of TbFab1 is

233 present as a $63 \mathrm{kDa}$ species (Fig. 3, asterisk), although a full length species was specifically

234 detected in the transformed cell line (Fig. 3, arrowhead). In addition, minor amounts of specific

235 polypeptides were consistently seen in the 50-100 kDA range, presumably the result of

236 degradation. Published transcriptomic datasets $[47,48]$ and our own RT-PCR analyses (data

237 not shown) indicate that the full-length TbFab1 transcript is present. However, these

238 immunoblot analyses indicate that the $156 \mathrm{kDa}$ isoform constitutes only a tiny fraction of total

239 steady state TbFab1.

240 Immunofluorescence analysis (IFA) of the TbFab1-HA cell line, costained for the

241 lysosomal marker TbCatL, indicated that TbFab1-HA localizes in the post-nuclear region,

242 consistent with a role in endocytic trafficking. A mixture of profiles was observed ranging from

243 prominent to weak colocalization with the lysosome (Fig. 4A, left to right). This peri-lysosomal

244 localization suggests that TbFab1 may also be present on late endosomes, which are closely

245 associated with the lysosome in T. brucei [14]. To address this we Ty epitope-tagged one

246 chromosomal allele of TbRab7, a late endosomal marker, in the TbFab1-HA cell line.

247 Costaining for the HA and Ty epitopes consistently showed overlapping localization (Fig. 4B).

248 Collectively these results indicate that TbFab1 localizes to the late endo/lysosomal membranes.

$249 \quad$ Fluorescent biosensors consisting of protein domains that bind specific PIPs fused to

250 GFP have been used to localize PIPs in many cell types [49]. The ENTH domain of yeast Ent3p

251 has been shown to specifically bind $\mathrm{PI}(3,5) \mathrm{P} 2$ in vitro [36]. We expressed this biosensor domain

252 with a 3xHA epitope tag ectopically in T. brucei and used IFA to localize the reporter.

253 Immunoblots with anti-HA indicated expression of tagged sensor of the expected size ( $\sim 26 \mathrm{kDa}$, 
254 not shown). Costaining with antibodies to TbCatL revealed a more restricted localization in the

255 lysosome than seen for TbFab1-HA (Fig. 4C). Together, these results support a role for

$256 \mathrm{TbFab1}$ in the late endosome/lysosome, but suggest that its product, $\mathrm{PI}(3,5) \mathrm{P}_{2}$

257 . accumulates most prominently at the lysosome.

$259 \quad 3.3$ TbFab1 is dispensable for endocytic and biosynthetic lysosomal trafficking The

260 ocalization of TbFab1 and the profound growth defect we observed when TbFab1 is knocked

261 down suggests that TbFab1 may function in endo-lysosomal trafficking. To address this, we

262 used a set of standard assays to test various routes to the lysosome. First, we measured the

263 ability of cells to take up Alexa488-tomato lectin (TL) as a surrogate for receptor-mediated

264 endocytosis. TL binds to N-linked carbohydrate epitopes in the flagellar pocket and is efficiently

265 taken up and delivered to the lysosome [14, 50]. Control and TbFab1 knockdown cells were

266 incubated with $\mathrm{TL}$ at $4^{\circ} \mathrm{C}$, to measure binding to the flagellar pocket, or $37^{\circ} \mathrm{C}$, to measure

267 endocytosis, followed by analysis by flow cytometry (Fig. 5A). In both cases, mean cell

268 associated fluorescence was equivalent in the two populations, indicating that TbFab1 is not

269 required for endocytosis. Next, we tested whether TbFab1 is required for delivery of

270 endocytosed material to the lysosome. Cells were incubated with FITC-TL for 30 minutes at

$2714^{\circ} \mathrm{C}$, then washed and shifted to $37^{\circ} \mathrm{C}$ to allow endocytosis (Fig. 5B). Because FITC

272 fluorescence is $\mathrm{pH}$ sensitive the fluorescence signal decreases as $\mathrm{TL}$ traverses the increasingly

273 acidic endosomal compartments to the terminal lysosome [51]. No difference was seen in the

274 rate or extent of signal loss indicating that transport of receptor-bound cargo to the lysosome

275 was not affected by TbFab1 depletion. The equivalent decline in FITC fluorescence also

276 suggests that lysosomes of TbFab1-knockdown cells have a normal internal $\mathrm{pH}$.

277 Trafficking of biosynthetic cargo to the lysosome can be measured by using pulse-chase

278 analysis to monitor the processing of the endogenous soluble lysosomal thiol protease TbCatL 
$279[14,52]$. TbCatL is synthesized as 53 and $50 \mathrm{kDa}$ proproteins ("I" and "X," respectively) that are

280 processed to a $44 \mathrm{kDa}$ mature form ("M") upon arrival in the lysosome. Knockdown had no

281 affect on the rate of TbCatL processing (Fig. 5C), indicating that TbFab1 is dispensable for

282 biosynthetic trafficking to the lysosome.

283

2843.4 TbFab1 is required for turnover of ISG65 ISG65 (invariant surface glycoprotein 65 kDa)

$285[53,54]$ is a type I membrane glycoprotein with lysine residues in its cytoplasmic tail that can be 286 ubiquitinylated to mark the protein for degradation in the lysosome [21-23]. Ubiquitin-targeted 287 degradation is mediated by the ESCRT machinery in other systems, and such has been argued 288 for ISG65 turnover in trypanosomes [20]. However, work from our laboratory has called this into 289 question, suggesting rather that the ESCRT machinery is actually involved in rescue of ISG65 290 from lysosomal degradation [15]. Given these different interpretations, and the fact that $291 \mathrm{PI}(3,5) \mathrm{P}_{2}$ has been implicated in ESCRT function in other systems [26, 27, 55], we asked what 292 role TbFab1 might play in regulating ISG65 turnover.

293 A cycloheximide-chase protocol [15] was used to measure the turnover of ISG65 in 294 control and TbFab1-knockdown cells (Fig. 6). In control cells, $\sim 60 \%$ of initial steady state 295 ISG65 was degraded during the 4-hour chase period, and this degradation was almost 296 completely blocked by treatment of cells with the thiol protease inhibitor FMK024, confirming

297 turnover in the lysosome. Silencing of TbFab1 completely eliminated ISG65 turnover, 298 demonstrating that TbFab1 is required for efficient targeting to the lysosome for degradation.

$300 \quad 3.5$ TbFig4 is required for ISG65 turnover In yeast, strains with Fig4 mutations typically have 301 the same phenotype as those with Fab1 mutations, likely because the entire Fab1:Vac14:Fig4 302 complex is required for Fab1 activity [41]. To address whether this might also be the case in $T$. 303 brucei, we expressed a tet-inducible TbFig4 RNAi construct in BSF cells. Cell growth was 
304 normal for the first $24 \mathrm{hrs}$ of silencing and thereafter was significantly retarded (Fig. 7A). TbFig 4

305 mRNA levels were reduced $\sim 50 \%$ at $24 \mathrm{hrs}$ (qRT-PCR, $\%$ of control $53.7 \pm 5.6$, mean \pm S.D., $n=3$ )

306 and ISG65 turnover was completely abolished (Fig. 7B). The phenocopying of TbFab1 and

307 TbFig4 silencing are consistent with function in the same pathway and the existence of a

308 complex as seen in other systems.

310 4. Discussion

311 Recent studies have advanced our understanding of the role of the late endosome in

312 lysosomal trafficking in T. brucei, revealing significant contrasts between trypanosomes and

313 other model systems. However, detailed knowledge is still lacking in trypanosomes, prompting

314 our investigation of $\mathrm{PI}(3,5) \mathrm{P}_{2}$, a potent signaling molecule with many roles in late

315 endo/lysosomal trafficking. Biochemical analyses indicate that $\mathrm{PI}(4,5) \mathrm{P}_{2}$ is the major $\mathrm{PIP}_{2}$

316 species in trypanosomes, but $\mathrm{PI}(3,5) \mathrm{P} 2$ is a small but detectable portion of the total. These

317 esults agree with similar measurements in yeast and in animals cells in which $\mathrm{PI}(3,5) \mathrm{P}_{2}$ is

318 present at as little as $1 \%$ the level of $\mathrm{PI}(4,5) \mathrm{P}_{2}[37,43-45]$. A previous study also identified

$319 \mathrm{PI}(4,5) \mathrm{P}_{2}$ as the major $\mathrm{PIP}_{2}$ species in trypanosomes using methodology that may not have had

320 the sensitivity required to detect low levels of $\mathrm{PI}(3,5) \mathrm{P}_{2}$ [56]. Consequently, this is the first

321 formal demonstration of $\mathrm{PI}(3,5) \mathrm{P}_{2}$ in trypanosomes. Hypotonic stress upregulates $\mathrm{PI}(3,5) \mathrm{P}_{2}$

322 levels in yeast, and this proved to be a useful strategy in validating our HPLC assay. It is

323 presently unknown whether stress conditions or developmental changes may similarly lead to

324 upregulation of $\mathrm{PI}(3,5) \mathrm{P}_{2}$ in $T$. brucei.

325 Simple homology searches identified clear orthologues of the ternary complex that

326 regulates $\mathrm{PI}(3,5) \mathrm{P}_{2}$ metabolism: Fab1 (kinase), Fig4 (phosphatase), Vac14 (scaffold). RNAi

327 silencing of TbFab1 resulted in a rapid and persistent cessation of growth followed by cell death

328 correlating with a significant reduction in the steady state level of $\mathrm{PI}(3,5) \mathrm{P}_{2}$. Based on this result

329 we conclude that TbFab1 is correctly identified as a PI3P-5 kinase. C-terminal chromosomal 
330 epitope tagging was used to characterize the TbFab1 protein. A full-length species (156 kDa)

331 was detected, but far and away the strongest signal was a $63 \mathrm{kDa}$ species. Trans-splicing

332 datasets indicate alternate use of an internal splice acceptor site that would be consistent with a

333 truncated TbFab1 of this size [46]. Interestingly, this truncation results in loss of three of four

334 homology domains in the TbFab1 orf (see Fig 1), most notably the FYVE PI3P binding domain.

335 Although the C-terminal catalytic domain remains, it is difficult to imagine this species playing a

336 major role in $\mathrm{PI}(3,5) \mathrm{P}_{2}$ metabolism in the absence of substrate recognition and membrane

337 binding. Rather, we assume that the full-length protein is the sole functional isoform.

338 Immunolocalization studies indicate variable colocalization of TbFab1 with the lysosome (p67),

339 and consistently strong overlapping localization with the late endosome (TbRab7). Interestingly,

340 when $\mathrm{PI}(3,5) \mathrm{P}_{2}$ was probed using an ENTH biosensor it was found to localize strongly with the

341 lysosome (TbCatL). These results suggest that the primary site of $\mathrm{PI}(3,5) \mathrm{P}_{2}$ synthesis is on late

342 endosomal, and to a lessor extent lysosomal, membranes, but that the end product resides

343 mostly in the lysosome. This is similar to the situation with $\mathrm{PI}(4,5) \mathrm{P}_{2}$, where the kinase

344 (TbPIPKA) localizes anterior to the mouth region of the flagellar pocket, but the product

345 localizes quite distinctly to the flagellar pocket proper [56].

346 Surprisingly, given all the known functions of $\mathrm{PI}(3,5) \mathrm{P}_{2}$ in other systems, knockdown of

347 TbFab1 had little effect on basic lysosomal trafficking. Uptake of endocytic cargo including

348 tomato lectin and transferrin (not shown) was unaffected. This contrasts with depletion of two

349 other PIP's, PI3P (TbVps34 knockdown) and PI(4,5)P 2 (TbPIPKA knockdown), both of which

350 result in defective endocytosis $[56,57]$, and further supports the specific assignment of TbFab1

351 activity to $\mathrm{PI}(3,5) \mathrm{P}_{2}$. In addition, lysosomal delivery, measured as decline in FITC fluorescence

352 as cargo moved to more acidic compartments, was unimpaired by TbFab1 depletion. This is

353 consistent with results from yeast, but different from observations in Drosophila and human cells,

354 where soluble cargo is readily taken up but fails to traffic to the lysosome $[58,59]$. This finding

355 also suggests that lysosomal $\mathrm{pH}$ is not affected by TbFab1 knockdown, as the final fluorescence 
356 intensity of control and knockdown cells was equivalent. Early studies of Fab1/PIKfyve-deficient

357 cells found elevated vacuolar/lysosomal $\mathrm{pH}[26,32]$. However, recent work using more

358 quantitative methods found that normal internal $\mathrm{pH}$ was maintained in the degradative

359 organelles of PIKfyve-inhibited mammalian or Fab1 $\Delta$ yeast cells [60], consistent with our

360 observations in T. brucei. Finally, TbFab1 ablation had no effect on biosynthetic trafficking to

361 the lysosome as assessed by the kinetics of TbCatL maturation, similar to results with

362 Cathepsin D in mammalian cells using conditional expression of a dominant negative PIKfyve

363 mutant [59].

364 The major phenotypic effect of TbFab1 knockdown is complete inhibition of lysosomal

365 degradation of ISG65, and this effect is recapitulated by knockdown of TbFig4. One might

366 expect that depletion of the $\mathrm{PI}(3,5) \mathrm{P}_{2}$ phosphatase would have the opposite effect, but in yeast

367 Fig4p is an essential member of the $\mathrm{PI}(3,5) \mathrm{P}_{2}$-regulating ternary complex and is required for

368 Fab1p activity [41]. Recent observations in mice suggest the same holds true in mammalian

369 systems [61]. Trypanosomes have orthologues of the all the components of the $\mathrm{PI}(3,5) \mathrm{P}_{2}$

370 complex, and it seems likely that a similar phenomenon is in play - knockdown of TbFig4

371 negatively impacts TbFab1 activity and hence the counter-intuitive phenocopying of TbFab1

372 knockdown. Collectively these results suggest that fine tuning of $\mathrm{PI}(3,5) \mathrm{P}_{2}$ levels is critical for

373 efficient regulation of ISG65 turnover.

374 The likely locus for $\mathrm{PI}(3,5) \mathrm{P}_{2}$ action is the LE/MVB compartment, which is marked by

375 TbRab7 and the ESCRT components TbVps23, TbVps28, and TbVps4 [14, 15, 20], and where

376 TbFab1 prominently localizes (this work). Our previous studies indicate that both TbRab7 and

377 TbVps4 act to retard ISG65 turnover, e.g., knockdown of either accelerates degradation, and

378 our favored model is that these agents facilitate retrieval/recycling from the LE/MVB to early

379 compartments of the endosomal system [15]. Whether this is dependent on unbiquitinylation is

380 not clear. Although the ESCRT machinery is typically thought to mediate lysosomal delivery, a

381 role in recycling is not unprecedented [24]. Within this hypothetical framework there are at least 
382 two obvious (simplistic) ways in which $\mathrm{PI}(3,5) \mathrm{P}_{2}$ might function in ISG65 trafficking. First,

$383 \mathrm{PI}(3,5) \mathrm{P}_{2}$ could be a negative regulator of Rab7/ESCRT-dependent rescue. In this scheme 384 depletion of $\mathrm{PI}(3,5) \mathrm{P}_{2}$ leads to enhanced recycling of ISG65 from the LE/MVB. Alternatively, $385 \mathrm{PI}(3,5) \mathrm{P}_{2}$ could be a positive regulator of a distinct Rab7/ESCRT-independent pathway for 386 LE/MVB-to-lysosome trafficking. In this scenario $\mathrm{PI}(3,5) \mathrm{P}_{2}$ depletion would block delivery of 387 ISG65 to the lysosome. Such a pathway, should it exist, would likely be distinct from normal 388 biosynthetic pathways to the lysosome since TbFab1 ablation has no effect on normal transport 389 of TbCatL.

$390 \quad$ In either scenario a complete understanding of how $\mathrm{PI}(3,5) \mathrm{P}_{2}$ mediates LE/MVB 391 pathways in trypanosomes will depend on identifying the relevant effector proteins it recruits.

392 One possibility is TbEpsinR [62], the sole ENTH domain protein evident in the T. brucei genome, 393 which shares significant homology with yeast Ent3p, a known $\mathrm{PI}(3,5) \mathrm{P}_{2}$ effector with a role in 394 sorting cargo into ILVs [36]. However, TbEpsin localizes to early endosomes and the flagellar 395 pocket, not the LE/MVB, where it functions in clathrin-dependent endocytosis. This localization 396 is dependent on synthesis of PI4P (PI4KIII $\beta$ knockdown), and presumably its derivative $397 \mathrm{PI}(4,5) \mathrm{P}_{2}$ [62]. Furthermore, as noted above, TbPIPKA, the kinase responsible for $\mathrm{PI}(4,5) \mathrm{P}_{2}$ 398 synthesis, localizes to the flagellar pocket mouth [56]. Taken together these data argue against 399 a role for TbEpsinR in trafficking pathways specific to the LE/MVB. A more promising effector protein in ISG65 sorting pathway(s) may be the ESCRT III 401 protein $\mathrm{Vps} 24$, which has been shown to bind $\mathrm{PI}(3,5) \mathrm{P}_{2}$ in mammalian cells [63]. TbVps24 402 (Tb927.11.10000) has not been studied empirically, but as a putative ESCRT III component it 403 would be expected to play a role in vesicle scission into the lumen of late endosomes. A 404 mechanism could be envisioned whereby negative regulation of ESCRT-dependent ISG65 405 rescue is mediated by $\mathrm{PI}(3,5) \mathrm{P}_{2}$ binding to TbVsp24. Obviously, there are even more 406 speculative and complex scenarios that could be elaborated to account for the unusual behavior 407 of ISG65 in the LE/MVB compartment, and more work will be required to bring clarity, but 
408 collectively our findings lay a solid experimental foundation for future investigations. Such work

409 should be informative, both to the specifics of late endosomal trafficking in trypanosomes, and in

410 a broader sense to more complex eukaryotic systems.

411

412 Acknowledgements The authors are grateful to Professor Mark Carrington (Cambridge

413 University, UK) for anti-ISG65. This work was supported by United States Public Health Service

414 Grants R01 AI056866 to JDB and F32 AIAl104147 to JKG, and by the Bill and Melinda Gates

415 Foundation Grant OPP1126862 to JMM. ROC was supported by the CAPES

416 Foundation/Science Without Borders, Brazil. 


\section{References}

[1] A. Schwede, M. Carrington, Bloodstream form trypanosome plasma membrane proteins: antigenic variation and invariant antigens, Parasitol. 137 (2010) 2029-2039.

[2] G. Rudenko, African trypanosomes: the genome and adaptations for immune evasion, Essays Biochem. 51 (2011) 47-62.

[3] S.G. Langreth, A.E. Balber, Protein uptake and digestion in bloodstream and culture forms of Trypanosoma brucei, J. Protozool. 22 (1975) 40-53.

[4] G.W. Morgan, C.L. Allen, T.R. Jeffries, M. Hollinshead, M.C. Field, Developmental and morphological regulation of clathrin-mediated endocytosis in Trypanosoma brucei, J. Cell Sci. 114 (2001) 2605-2015.

[5] A.E. Balber, The pellicle and the membrane of the flagellum, flagellar adhesion zone, and flagellar pocket: functionally discrete surface domains of the bloodstream form of African trypanosomes, Crit. Rev. Immunol. 10 (1990) 177-201.

[6] J.D. Barry, Capping of variable antigen on Trypanosoma brucei, and its immunological and biological significance, J. Cell Sci. 37 (1979) 287-302.

[7] A. Pal, B.S. Hall, T.R. Jefferies, M.C. Field, Rab5 and Rab11 mediate transferrin and antivariant surface glycoprotein recycling in Trypanosoma brucei, Biochem. J. 374 (2003) 443-451.

[8] M. Engstler, T. Pfohl, S. Herminghaus, M. Boshart, G. Wiegertjes, N. Heddergott, P. Overath, Hydrodynamic flow-mediated protein sorting on the cell surface of trypanosomes, Cell 131 (2007) 505-515.

[9] S. Alsford, S. Eckert, N. Baker, L. Glover, A. Sanchez-Flores, K.F. Leung, D.J. Turner, M.C. Field, M. Berriman, D. Horn, High-throughput decoding of antitrypanosomal drug efficacy and resistance, Nature 482 (2012) 232-236.

[10] K.M. Hager, M.A. Pierce, D.R. Moore, E.M. Tytler, J.D. Esko, S.L. Hajduk, Endocytosis of a cytotoxic human high density lipoprotein results in disruption of acidic intracellular vesicles and subsequent killing of African trypanosomes, J. Cell Biol. 126 (1994) 155-167.

[11] M. Engstler, L. Thilo, F. Weise, C.G. Grünfelder, H. Schwarz, M. Boshart, P. Overath, Kinetics of endocytosis and recycling of the GPI-anchored variant surface glycoprotein in Trypanosoma brucei, J. Cell Sci. 117 (2004) 1105-1115.

[12] B. Hall, C.L. Allen, D. Goulding, M.C. Field, Both of the Rab5 subfamily of small GTPases of Trypanosoma brucei are essential and required for endocytosis., Mol. Biochem. Parasitol. 138 (2004) 67-77.

[13] T.R. Jefferies, G.W. Morgan, M.C. Field, A developmentally regulated rab11 homologue in Trypanosoma brucei is involved in recycling processes, J. Biol. Chem. 114 (2001) 2617-2626.

[14] J.S. Silverman, K.J. Schwartz, S.L. Hajduk, J.D. Bangs, Late endosomal Rab7 regulates lysosomal trafficking of endocytic but not biosynthetic cargo in Trypanosoma brucei, Mol. Microbiol. 82 (2011) 664-678. 
[15] J.S. Silverman, K.A. Muratore, J.D. Bangs, Characterization of the late endosomal ESCRT machinery in Trypanosoma brucei, Traffic 14 (2013) 1078-1090.

[16] K. Hagland, I. Dikic, The role of ubiquitylation in receptor endocytosis and endosomal sorting, J. Cell Sci. 125 (2012) 265-275.

[17] P.I. Hanson, A. Cashikar, Multivesicular body morphogenesis, Annu. Rev. Cell Dev. Biol. 28 (2012) 337-362.

[18] W.M. Henne, N.J. Buchkovich, S.D. Emr, The ESCRT pathway, Dev. Cell 21 (2011) 77-91.

[19] A. Shestakova, A. Hanono, S. Drosner, M. Curtiss, B.A. Davies, D.J. Katzmann, M. Babst, Assembly of the AAA ATPase Vps4 on ESCRT-III, Mol. Biol. Cell 21(6) (2010) 1059-71.

[20] K.F. Leung, J.B. Dacks, M.C. Field, Evolution of the multivesicular body ESCRT machinery; retention across the eukaryotic lineage, Traffic 9 (2008) 1698-716.

[21] W.-L. Chung, M. Carrington, M.C. Field, Cytoplasmic targeting signals in transmembrane invariant surface glycoproteins of trypanosomes, J. Biol. Chem. 279 (2004) 54887-54895.

[22] W.L. Chung, K.F. Leung, M. Carrington, M.C. Field, Ubiquitylation is required for degradation of transmembrane surface proteins in trypanosomes, Traffic 9(10) (2008) 1681-97.

[23] K.F. Leung, F.S. Riley, M. Carrington, M.C. Field, Ubiquitylation and developmental regulation of invariant surface protein expression in trypanosomes, Euk. Cell 10(7) (2011) 91631.

[24] A. Baldys, J.R. Raymond Sr, Critical role of ESCRT machinery in EGFR recycling, Biochem. 48 (2009) 9321-9323.

[25] F.T. Cooke, S.K. Dove, R.K. McEwen, G. Painter, A.B. Holmes, M.N. Hall, R.H. Michell, The stress-activated phosphatidyl 3-phosphate 5-kinase Fab1p is essential for vacuole function in S. cerevisiae, Curr. Biol. 8 (1997) 1219-1222.

[26] J.D. Gary, A.E. Wurmser, B.C. J, L.S. Weisman, S.D. Emr, Fab1p is essential for Ptdlns(3)P 5-kinase activity and the maintenance of vacuolar size and membrane homeostasis, J. Cell Biol. 143 (1998) 65-79.

[27] S.K. Dove, R.K. McEwen, A. Mayes, D.C. Hughes, J.D. Beggs, R.H. Michell, Vac14 controls Ptdlns(3,5) P(2) synthesis and Fab1-dependent protein trafficking to the multivesicular body, Curr. Biol. 12 (2002) 885-893.

[28] S.A. Rudge, D.M. Anderson, S.D. Emr, Vacuole size control: regulation of Ptdlns(3,5)P2 levels by the vacuole-associated Vac14-Fig4 complex, a Ptdlns(3,5)P2-specific phosphatase, Mol. Biol. Cell 15 (2004) 24-36.

[29] A.-S. Nicot, H. Fares, B. Payrastre, A.D. Chisholm, M. Labouesse, J. Laporte, The phosphoinositide kinase PIKfyve/Fab1p regulates terminal lysosome maturation in Caenorhabditis elegans, Mol. Biol. Cell 17 (2006) 3062-3074. 
[30] T.E. Rusten, L.M.W. Rodahl, K. Pattni, C. Englund, C. Samakovlis, S. Dove, A. Brech, H. Stenmark, Fab1 phosphatidylinositol 3-phosphate 5-kinase controls trafficking but not silencing of endocytosed receptors, Mol. Biol. Cell 17 (2006) 3989-4001.

[31] Y. Zhang, S.N. Zolov, C.Y. Chow, S.G. Slutsky, S.C. Richardson, R.C. Piper, B. Yang, J.J. Nau, R.J. Westrick, S.J. Morrison, M.H. Meisler, L.S. Weisman, Loss of Vac14, a regulator of the signaling lipid phosphatidylinositol 3,5-bisphosphate, results in neurodegeneration in mice, Proc. Natl. Acad. Sci. USA 104 (2007) 17518-17523.

[32] A. Yamamoto, D.B. DeWald, I.V. Boronenkov, R.A. Anderson, S.D. Emr, D. Koshland, Novel PI(4)P 5-kinase homologue, Fab1p, essential for normal vacuole function and morphology in yeast., Mol. Biol. Cell 6 (1995) 525-539.

[33] H. Hirumi, K. Hirumi, Axenic culture of African trypanosome bloodstream forms, Parasitol. Today 10 (1994) 81-84.

[34] E. Wirtz, S. Leal, C. Ochatt, G. Cross, A tightly regulated inducible expression system for conditional gene knockouts and dominant-negative genetics in Trypanosoma brucei, Mol. Biochem. Parasitol. 99 (1999) 89-101.

[35] D.J. LaCount, S. Bruse, K.L. Hill, J.E. Donelson, Double-stranded RNA interference in Trypanosoma brucei using head-to-head promoters., Mol. Biochem. Parasitol. 111 (2000) 67-76.

[36] S. Friant, E. Pécheur, A. Eugster, F. Michel, Y. Lefkir, D. Nourrisson, F. Letourneur, Ent3p Is a Ptdlns(3,5)P2 effector required for protein sorting to the multivesicular body, Dev. Cell 5 (2003) 499-511.

[37] D.R. Jones, I.B.-R. Ramireez, M. Lowe, N. Divecha, Measurement of phosphoinositides in the zebrafish Danio rerio, Nat. Protoc 8 (2013) 1058-1072.

[38] R.F. Peck, A.M. Shiflett, K.J. Schwartz, A. McCann, S.L. Hajduk, J.D. Bangs, The LAMPlike protein p67 plays an essential role in the lysosome of African trypanosomes, Mol. Microbiol. 68 (2008) 933-946.

[39] A.E. Gruszynski, A. DeMaster, N.M. Hooper, J.D. Bangs, Surface coat remodeling during differentiation of Trypanosoma brucei, J. Biol. Chem. 278 (2003) 24665-24672.

[40] N. Jin, C.Y. Chow, L. Liu, S.N. Zolov, R. Bronson, M.J. Davisson, J.L. Petersen, Y. Zhang, S. Park, J.E. Duex, D. Goldowitz, M.H. Meisler, L.S. Weisman, VAC14 nucleates a protein complex essential for the acute interconversion of $\mathrm{PI} 3 \mathrm{P}$ and $\mathrm{PI}(3,5) \mathrm{P}_{2}$ in yeast and mouse, EMBO J 27 (2008) 3221-3234.

[41] R.J. Botelho, J.A. Efe, D. Teis, S.D. Emr, Assembly of a Fab1 phosphoinositide kinase signaling complex requires the Fig4 phosphoinositide phosphatase, Mol. Biol. Cell 19 (2008) 4273-4286.

[42] A.J. McCartney, Y. Zhang, L.S. Weisman, Phosphatidylinositol 3,5-bisphosphate: Low abundance, high significance, BioEssays 36 (2013) 52-64. 
[43] C.J. Bonangelino, J.J. Nau, J.E. Duex, M. Brinkman, A.E. Wurmser, J.D. Gary, S.D. Emr, L.S. Weisman, Osmotic stress-induced increase of phosphatidylinositol 3,5-bisphosphate requires Vac14p, an activator of the lipid kinase Fab1p, J. Cell Biol. 156 (2002) 1015-1028.

[44] S.N. Zolov, D. Bridges, Y. Zhang, W.-W. Lee, E. Riehle, R. Verma, G.M. Lenk, K. Converso-Baran, T. Weide, R.L. Albin, A.R. Saltiel, M.H. Meisler, M.W. Russell, L.S. Weisman, In vivo, Pikfyve generates $\mathrm{PI}(3,5) \mathrm{P}_{2}$, which serves as both a signaling lipid and the major precursor for PI5P, Proc. Natl. Acad. Sci. USA 109 (2012) 17472-17477.

[45] S.K. Dove, F.T. Cooke, M.R. Douglas, L.G. Sayers, P.J. Parker, R.H. Michell, Osmotic stress activates phosphatidylinositol-3,5-bisphosphate synthesis, Nature 390 (1997) 187-192.

[46] D. Nilsson, K. Gunasekera, J. Mani, M. Osteras, L. Farinelli, L. Baerlocher, I. Roditi, T. Ochsenreiter, Spliced Leader Trapping Reveals Widespread Alternative Splicing Patterns in the Highly Dynamic Transcriptome of Trypanosoma brucei, PLoS Pathog. 6 (2010) e1001037.

[47] T.N. Siegel, D.R. Hekstra, X. Wang, S. Dewell, G.A.M. Cross, Genome-wide analysis of mRNA abundance in two life-cycle stages of Trypanosoma brucei and identification of splicing and polyadenylation sites. , Nucleic Acids Res. 38 (2010) 4946-4957.

[48] J.-J. Vasquez, C.-C. Hon, J.T. Vanselow, A. Schlosser, T.N. Siegel, Comparative ribosome profiling reveals extensive translational complexity in different Trypanosoma brucei life cycle stages., Dev. Cell 5 (2014) 3623-3637.

[49] G.R. Hammond, T. Balla, Polyphosphoinositide binding domains: Key to inositol lipid biology, Biochim. Biophys. Acta 1851 (2015) 746.

[50] A.K. McCann, K.J. Schwartz, J.D. Bangs, A determination of the steady state lysosomal pH of bloodstream stage African trypanosomes, Mol. Biochem. Parasitol. 159 (2008) 146-149.

[51] S. Ohkuma, B. Poole, Fluorescence probe measurement of the intralysosomal pH in living cells and the perturbation of pH by various agents, Proc. Natl. Acad. Sci. USA 75 (1978) 33273331.

[52] E.S. Sevova, J.D. Bangs, Streamlined architecture and GPI-dependent trafficking in the early secretory pathway of African trypanosomes, Mol. Biol. Cell 20 (2009) 4739-4750.

[53] K. Ziegelbauer, P. Overath, Identification of invariant surface glycoproteins in the bloodstream stage of Trypanosoma brucei, J. Biol. Chem. 267 (1992) 10791-10796.

[54] P. Overath, M. Chaudri, D. Steverding, K. Ziegelbauer, Invariant surface proteins in bloodstream forms of Trypanosoma brucei, Parasitol. Today 10 (1994) 53-58.

[55] G. Odorizzi, M. Babst, S. Emr, Fab1p Ptdlns(3)P 5-kinase function is essential for protein sorting in the multivesicular body, Cell 95 (1998) 847-858.

[56] L. Demmel, K. Schmidt, L. Lucast, K. Havicek, A. Zankel, T. Koestler, V. Reithofer, P. de Camilli, G. Warren, The endocytic activity of the flagellor pocket in Trypanosoma brucei is regulated by an adjacent phosphatidylinositol phosphate kinase., J. Cell Sci. 127 (2014) 23512364. 
[57] B.S. Hall, C. Gabernet-Castello, A. Voak, D. Goulding, S.K. Natesan, M.C. Field, TbVps34, the trypanosome orthologue of Vps34, is required for Golgi complex segregation, J. Biol. Chem. 281 (2006) 27600-27612.

[58] T.E. Rustin, T. Caccari, H. Stenmark, Shaping development with ESCRTs, Nat. Cell Biol. (14) (2012) 38-45.

[59] O.C. Ikonomov, D. Sbrissa, M. Foti, J.-L. Carpentier, A. Shisheva, PIKfyve controls fluid phase endocytosis but not recycling/degradation of endcytosed receptors or sorting of procathepsin D by regulating multivesicular body morphogenesis, Mol. Biol. Cell 14 (2003) 4581-4591.

[60] C.Y. Ho, C.H. Choy, C.A. Wattson, D.E. Johnson, R.J. Botelho, The Fab1/PIKfyve phosphoinositide phosphate kinase is not necessary to maintain the $\mathrm{pH}$ of lysosomes and of the yeast vacuole, J. Biol. Chem. 290 (2015) 9919-9928.

[61] G.M. Lenk, C.M. Frei, A.C. Miller, R.C. Wallen, Y.A. Mironova, R.J. Giger, M.H. Meisler, Rescue of neurodegeneration in the Fig4 mull mouse by a catalytically inactive FIG4 gene, Hum. Mol. Genet. 25 (2016) 340-347.

[62] C. Gabernet-Castello, J.B. Dacks, M.C. Field, The single ENTH-domain protein of trypanosomes; endcytic functions and evolutionary relationship with epsin, Traffic 10 (2009) 894-911.

[63] P. Whitely, B.J. Reaves, M. Hashimoto, A.M. Riley, B.V.L. Potter, G.D. Holman, Identification of mammalian Vps24p as an effector of phosphatidylinositol 3,5-bisphosphatedependent endosome compartmentalization, J. Biol. Chem. 278 (2003) 38786-38795. 


\section{Figure legends}

Figure 1. T. brucei Fab1 and Fig4 Domain structures of the T. brucei and S. cerevisiae Fab1 (Tb927.11.1460, AHY75806) and Fig4 (Tb927.11.5490, P42837) orthologues. Diagrams are to scale and sizes (amino acids residues) are in parentheses. The positions in TbFab1 of the alternate internal trans-splice site (asterisk) and of the introduced HA tag are indicated. Domains are: FYVE (PI3P binding); CCT (homology to chaperone Cpn60/TCP-1, Vac14 binding); CCR (conserved cysteine rich, Vac14 binding); Kinase (catalytic site); Sac (phosphoinositide phosphatase) [42].

Figure 2. $\mathrm{PI}(3,5) \mathrm{P}_{2}$ is dependent on $T b F a b 1$ expression $\mathrm{A}$. T. brucei and osmotically shocked S. cerevisiae were metabolically labeled overnight with $\left[{ }^{3} \mathrm{H}\right]$-myo-inositol and PIPs were extracted, saponified, and resolved by anion exchange HPLC. A full chromatograph is presented on the left, and an expansion of the $\mathrm{gPIP}_{2}$ region is on the right. Characteristic peaks corresponding to $\mathrm{gPIP}, \mathrm{gPI}(3,5) \mathrm{P}_{2}$ and $\mathrm{PI}(4,5) \mathrm{P}_{2}$ are indicated. Data are presented as the fraction of the total loaded sample. B. Growth of TbFab1 RNAi cells was evaluated by seeding at $10^{5} / \mathrm{mL}$ in media with or without tetracycline. Cell density was determined every 24 hours by hemocytometer and cultures were adjusted to starting density daily. Mean and standard deviation of 3 biological replicates is displayed. Data is representative of 3 independent experiments. C. TbFab1 RNAi cells were labeled overnight with $\left[{ }^{3} \mathrm{H}\right]$ myo-inositol with or without tetracycline induction and extracted gPIPs were resolved by HPLC (left) as in Panel A. Relative $\mathrm{PI}(3,5) \mathrm{P}_{2}$ levels were quantified (right) by normalizing to the $\mathrm{PI}(4,5) \mathrm{P}_{2}$ level in each sample (mean $\pm \mathrm{SD}, n=3, \mathrm{p}<0.02$ by unpaired $t$-test). Total loaded radiolabeled samples varied experimentally, but was typically $20,000-30,000 \mathrm{cpm}$ for both control and silenced cells

Figure 3. Expression of HA epitope tagged TbFab1 Parental (Par) and Fab1:HA cells (10 ${ }^{7}$ cell equivalents/lane) were subjected to immunoblotting with anti-HA antibody (left). Mobilities of the dominant short (asterisk, $63 \mathrm{kDa}$ ) and full length (arrowhead, $156 \mathrm{kDa}$ ) isoforms are indicated (asterisk). A separate blot was performed with anti-Hsp70 as a loading control (right). Mobilities of molecular mass markers $(\mathrm{kDa})$ are indicated.

Figure 4. TbFab1 and PI(3,5)P2 localize to the lysosome and late endosome IFA was performed with fixed permeabilized cells as described in Materials and Methods. Cells were stained with DAPI to reveal nuclei $(n)$ and kinetoplasts $(k)$ as indicated (left panels only). A. TbFab1-HA cells costained with antibodies against HA (green) and endogenous TbCatL (red), a lysosomal marker. B. TbFab1-HA cells co-expressing Ty-Rab7, a late endosome marker, stained with antibodies against HA (green) and Ty (red). C. Cells expressing ENTH-HA, a $\mathrm{PI}(3,5) \mathrm{P}_{2}$ biosensor, stained with antibodies against $\mathrm{HA}$ (green) and TbCatL (red). Each set contains three independent triple channel merges with magnified insets of single red, single green and merged red/green channel images of the post-nuclear region.

Figure 5. Effect of TbFab1 silencing on lysosomal trafficking TbFab1 RNAi cells were cultured with or without tetracycline for 18 hours prior to all assays. A. Cells were incubated (30 min) with A488-TL at $4^{\circ} \mathrm{C}$ (binding) or $37^{\circ} \mathrm{C}$ (uptake) and analyzed by flow cytometry. Data are presented as mean fluorescent intensity (MFI, mean $\pm S D, n=3$, representative of 3 independent experiments). B. Cells were incubated (30 min) with FITC-TL at $4^{\circ} \mathrm{C}$, washed, and then shifted to $37^{\circ} \mathrm{C}$ to allow endocytosis and trafficking to the lysosome. Analysis was by flow cytometry and the data are normalized to $T_{0}$ (mean $\pm S D, n=3$, representative of 3 independent experiments). C. Cells were pulse-chase radiolabeled and at the indicated times TbCatL was immunoprecipitated from cell lysates and fractionated by SDS-PAGE. A representative phosphorimage is presented with precursor $(\mathrm{I}, \mathrm{X})$ and mature $(\mathrm{M})$ polypeptides indicated 
(bottom). Loss of precursors and appearance of mature form were quantified and are presented graphically (top, mean $\pm S D, n=2$ ).

Figure 6. TbFab1 controls ISG65 turnover TbFab1 silencing was induced with tetracycline for 18 hours and then for an additional 4 hours with and without cycloheximide $(100 \mu \mathrm{g} / \mathrm{ml})$ as indicated. Duplicate cultures were treated with FMK024 $(20 \mu \mathrm{M})$ to inhibit degradation by lysosomal thiol proteases. Samples were prepared for immunoblotting with anti-ISG65 at $T_{0}$ and $\mathrm{T}_{4}$ of the second incubation. The membrane was stripped and reprobed with anti-BiP as a loading control. Representative blots are presented (top). ISG65 signals were quantified and normalized to BiP and are presented as the fraction of $\mathrm{T}_{0}$ for each data set (bottom, $n=3$ ).

Figure 7. TbFig4 A. Growth of TbFig4 RNAi cells was evaluated by seeding at $105 / \mathrm{mL}$ in media with or without tetracycline. Cell density was determined every 24 hours by hemocytometer and cultures were adjusted to starting density daily. Mean and standard deviation of 3 biological replicates is displayed. B. TbFig4 silencing was induced with tetracycline for 18 hours and then for an additional 4 hours with and without cycloheximide (100 $\mu \mathrm{g} / \mathrm{ml})$ as indicated. Duplicate cultures were treated with FMK024 $(20 \mu \mathrm{M})$ to inhibit degradation by lysosomal thiol proteases. Samples were prepared for immunoblotting with anti-ISG65 at $T_{0}$ and $\mathrm{T}_{4}$ of the second incubation. The membrane was stripped and reprobed with anti-BiP as a loading control. Representative blots are presented (left). ISG65 signals were quantified and normalized to BiP and are presented as the fraction of To for each data set (right, $n=3$ ). 

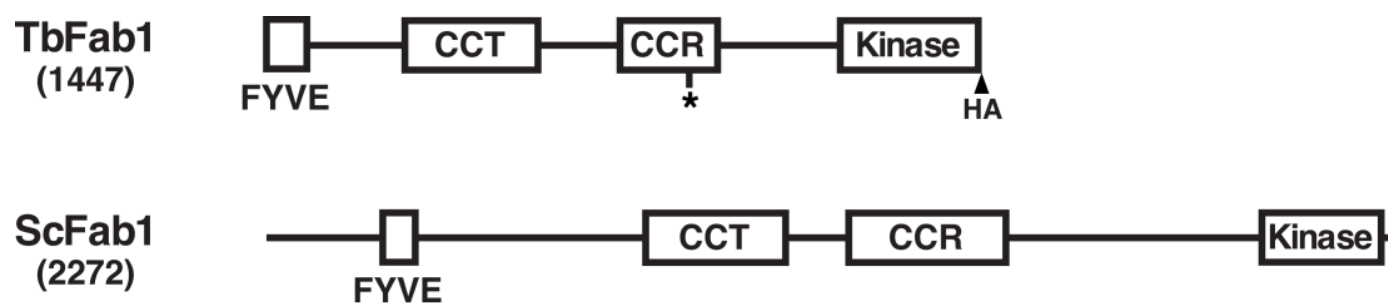

TbFig4

(1561)

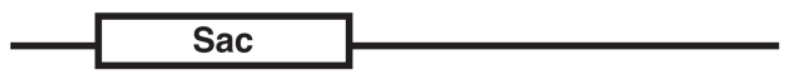

ScFig4

(879)

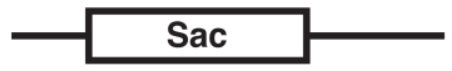



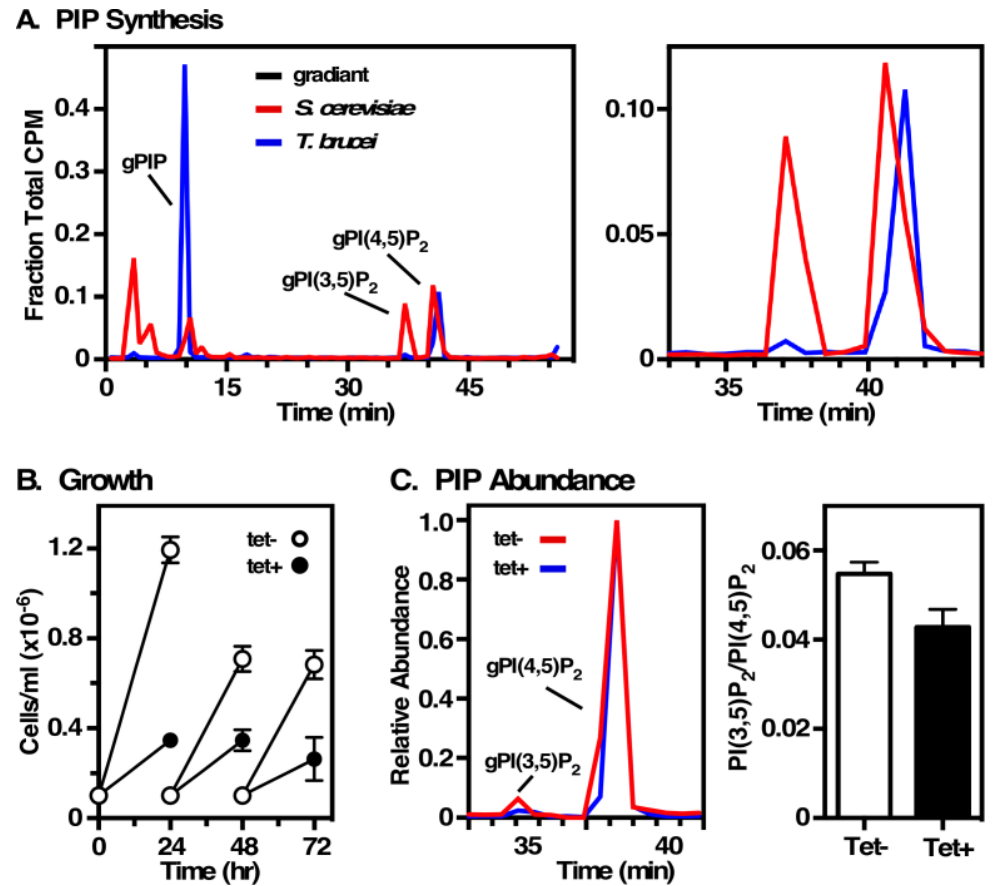


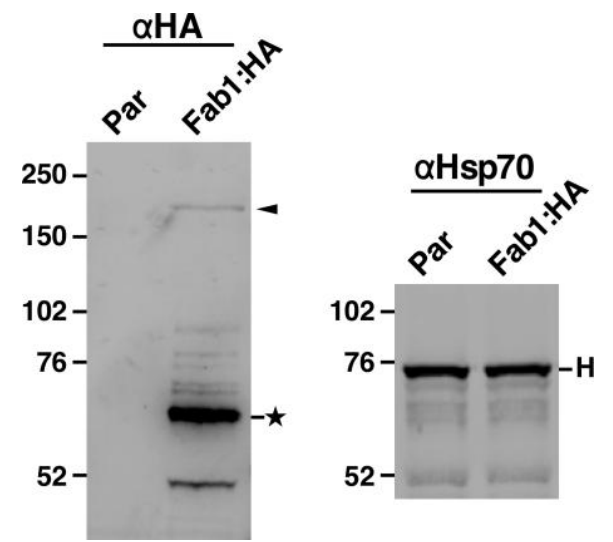


A. CatL vs TbFab1-HA

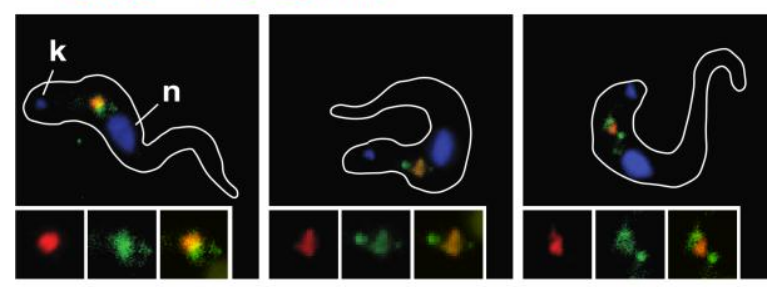

B. Ty-Rab7 vs TbFab1-HA

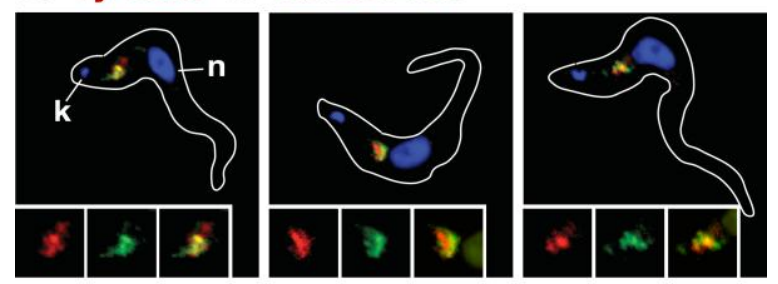

C. CatL vs ENTH-HA

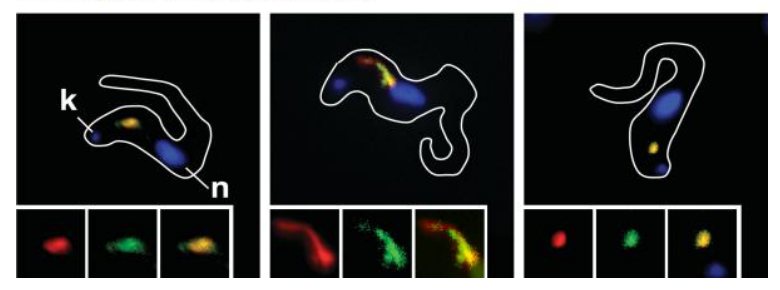


A. Endocytosis

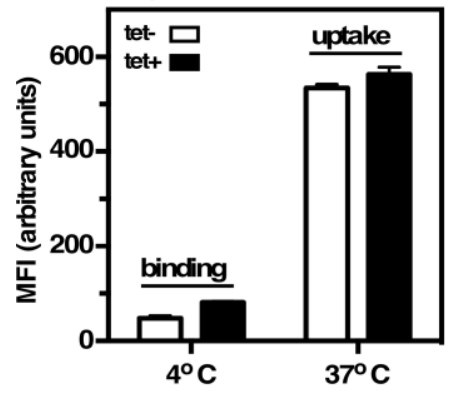

B. Lysosomal Delivery

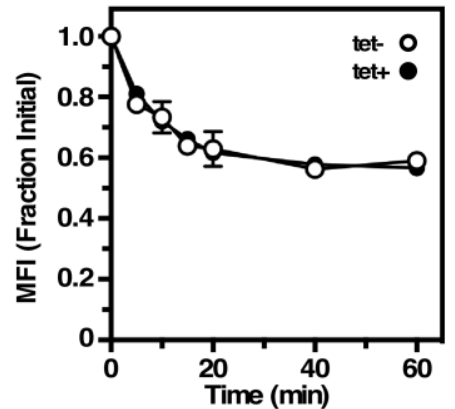

C. TbCatL
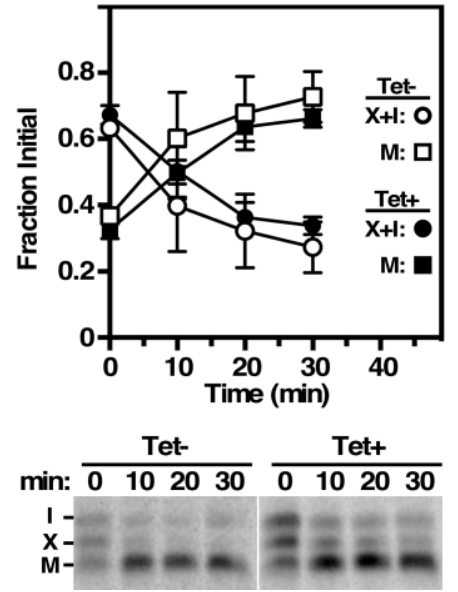

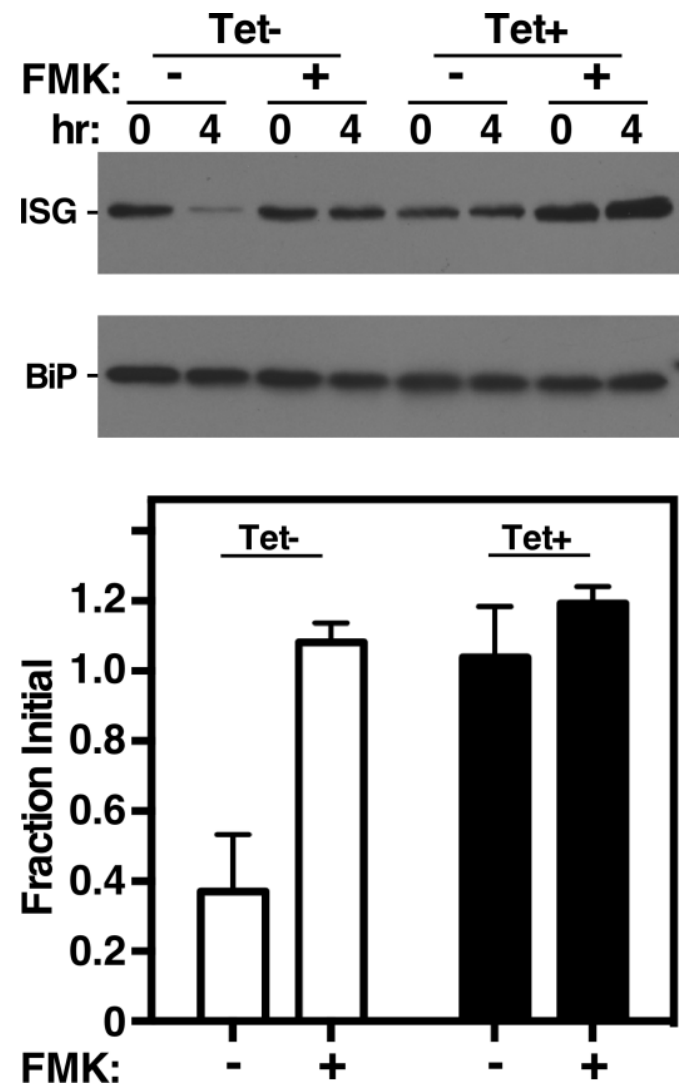
A. Growth

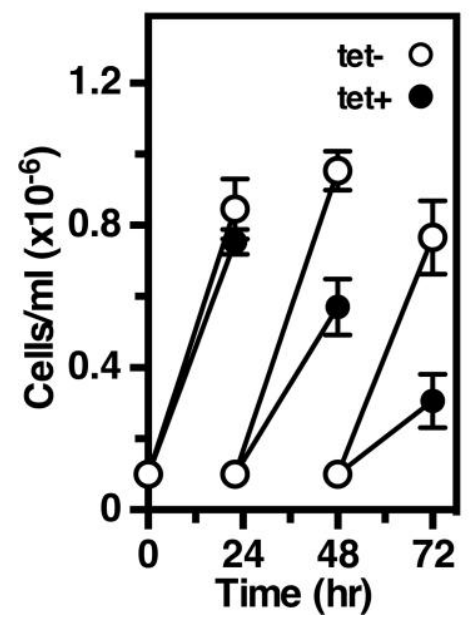

B. ISG65 Turnover

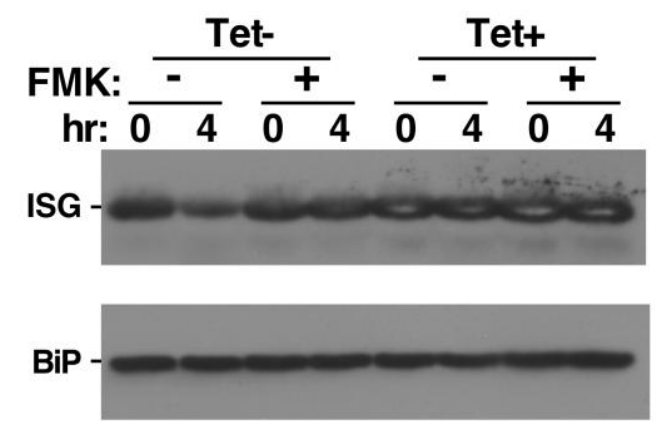

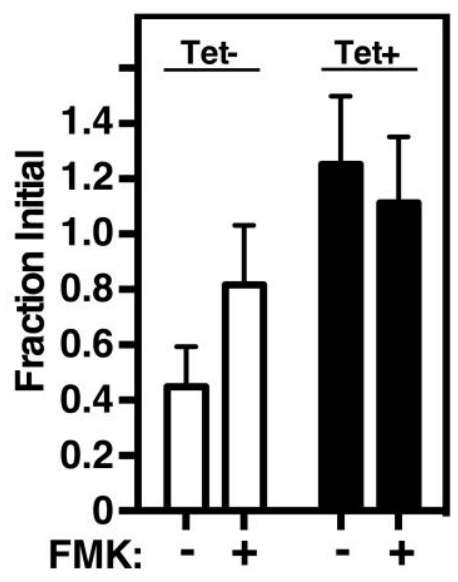

